\title{
Enhanced Terahertz Detection using Multiple GaAs HEMTs Connected in Series
}

\author{
Tamer A. Elkhatib ${ }^{1}$, Dmitry B. Veksler ${ }^{1}$, Khaled N. Salama ${ }^{2}$, Xi-C. Zhang ${ }^{1}$, and Michael S. Shur ${ }^{1}$ \\ ${ }^{1}$ Center for Terahertz Research, Rensselaer Polytechnic Institute, 110, 8th Street, Troy, NY, 12180, USA \\ ${ }^{2}$ Electrical Engineering Program, King Abdullah University of Science and Technology, Saudi Arabia
}

\begin{abstract}
We report here, for the first time, on enhanced nonresonant detection of terahertz radiation using multiple InGaAs/GaAs high-electron-mobility transistors (HEMTs) connected in series and biased by a direct drain current. A 1.63 $\mathrm{THz}(184 \mu \mathrm{m})$ response is proportional to the number of detecting transistors operating in saturation region at the same gate-source bias voltage. The experimental data are in agreement with the detection mechanism based on the rectification of overdamped plasma waves excited by radiation in channels of devices.

Index Terms- GaAs HEMTs, series transistors, room temperature, plasma waves, short channel, terahertz detection.
\end{abstract}

\section{INTRODUCTION}

Terahertz applications such as identification of drugs and explosives materials [1], medical diagnostics [2], and security imaging [3] stimulated rapid development of terahertz science and technology. $\mathrm{THz}$ detectors with fast response time are required for raster-scan imaging currently used in most existing terahertz imaging systems. The most common terahertz detectors available now are bolometers [4], pyroelectric detectors, Schottky diodes [5], and photoconductive detectors [6]. THz plasma wave oscillators [7]-[8], and detectors based on GaAs [9], GaN [10], $\mathrm{Si}$ [11], and silicon on insulator (SOI) [12] FETs have been reported. Some of these detectors demonstrated room temperature performance that is comparable to commercially available pyroelectric detectors in terms of sensitivity [13], but with a much higher speed of operation [14]-[15].

Since the first observation of plasma wave detection in short channel FETs [16], only single transistor structures (some with multiple gates) have been investigated. However, our theoretical analysis predicts dramatic enhancement of the detection responsivity in transistor arrays [17]-[18]. We now report on a multiple transistors design, in which symmetrical transistors are connected in series. Gates of all the transistors are biased separately. Drain current is driven through a battery and a load resistor to enhance the responsivity of terahertz detection [19]. We compare here the terahertz response from single transistor, two transistors, three transistors, and four transistors connected in series. Our experimental results show that detected terahertz response is proportional to the number of transistors connected in series. It also supports the conclusion that detection mechanism is based on the rectification of overdamped plasma waves excited in transistor channel [20].

\section{Multiple Transistors Structure}

Figure 1 presents die photos of the fabricated test structures of two transistors and four transistors connected in series. The $0.5 \mu \mathrm{m}$ enhancement-mode InGaAs/GaAs HEMT structures were fabricated by TriQuint Semiconductor. The main advantage of this technology is that it has a relatively high breakdown voltage that allows us to bias several transistors connected in series and guarantee that all of them operate in saturation region for the enhanced detection [21].

In order to test terahertz response from our multiple transistors design, radiation from a terahertz gas laser (SIFIR-50) was focused and incident normally on our test structures using the experimental setup shown in Fig. 2. Focusing of the $1.63 \mathrm{THz}$ laser beam was adjusted by mounting our test structures chip on a computer controlled three dimension nanopositioning stage (NanoMax 341) from ThorLabs. The terahertz laser beam waist was measured and found to be approximately $140 \mu \mathrm{m}$ (FWHM), while the areas of our multiple transistors design were $85 \times 32 \mu \mathrm{m}^{2}$ for the two transistors structure and $85 \times 50 \mu \mathrm{m}^{2}$ for the four transistors structure.
$S g_{1} g_{2} D$

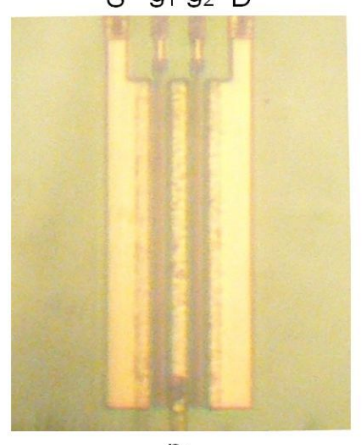

$\mathrm{n}_{1}$

(a)

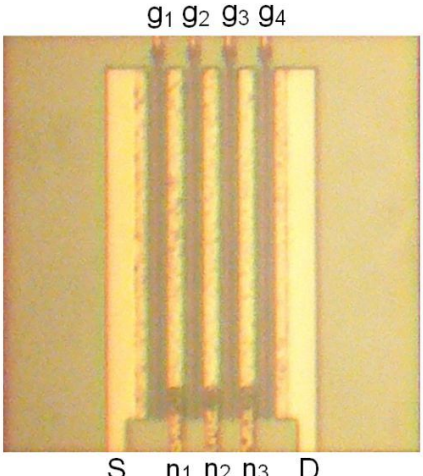

(b)
Fig. 1. Die photos of the fabricated test structures of (a) two transistors and (b) four transistors connected in series. The channel length and width of all transistors are $0.5 \mu \mathrm{m}$ and $80 \mu \mathrm{m}$ respectively. The separation distance between all gates is chosen to be $8 \mu \mathrm{m}$ to be much smaller than the terahertz wavelength. All terminals are labeled showing source $(S)$, drain (D), gates $\left(g_{1}, . ., g_{4}\right)$, and intermediate nodes $\left(\mathrm{n}_{1}, . ., \mathrm{n}_{3}\right)$. 


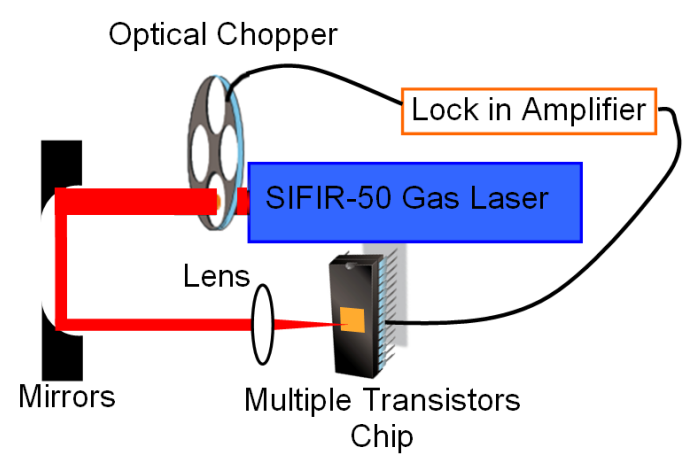

Fig. 2. Experimental setup for measuring terahertz response from our multiple transistors structure.

\section{EXPERIMENTAL RESULTS AND DISCUSSION}

We have tested our two transistors structure for terahertz detection in three different operating configurations. These configurations were: one transistor operated in saturation region while the other one was floating; one transistor operated in linear region and the second transistor (connected in series) operated in saturation region; and finally both transistors connected in series and operated in saturation region. Figure 3 presents the circuit schematics of the selected operating configurations showing the dc operating point at each node and dc biasing through a load resistor [22]; it also summarizes the measured responsivity for each of these configurations. The gate biasing was tuned such that each transistor detected with maximum responsivity.

Since the fraction of terahertz laser radiation coupled to our multiple transistors structure is not known precisely, the measured responsivity plots are related to terahertz laser power in arbitrary units. However, we measured the maximum power of the focused terahertz laser beam and it was found to be $35 \mathrm{~mW}$. Based on this power reading, the responsivity of our two transistors structure biased as shown in Fig. 3(a) and Fig. 3(b) is estimated to be $7 \mathrm{~V} / \mathrm{W}$. while the responsivity is doubled and equal to $14 \mathrm{~V} / \mathrm{W}$ when both transistors are operating in saturation region under the same gate-source biasing voltage as shown in Fig. 3(c). These estimates do not consider the much smaller area of our test structure relative to the focused laser beam waist. By considering the area ratio of the focused laser beam waist to that of each transistor in our test structure, the responsivity increases to $170 \mathrm{~V} / \mathrm{W}$ independent of the number of transistors used.

The interpretation of these results is in good agreement with plasma wave $\mathrm{THz}$ detection theory developed in [20]. According to the mechanism described in [20], overdamped plasma waves induced by the incident radiation in the channel of FET are rectified. This results in an induction of a dc voltage between the source and the drain terminals of the transistor, i.e. the $\mathrm{THz}$ response. This $\mathrm{THz}$ response can be tuned by the gate and drain biases controlling electron density in the channel.

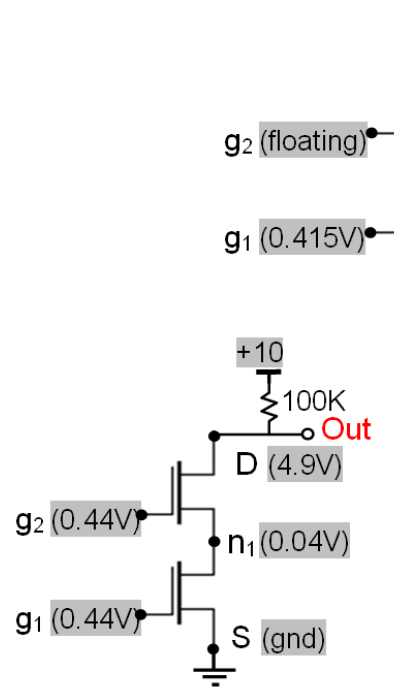

(b)

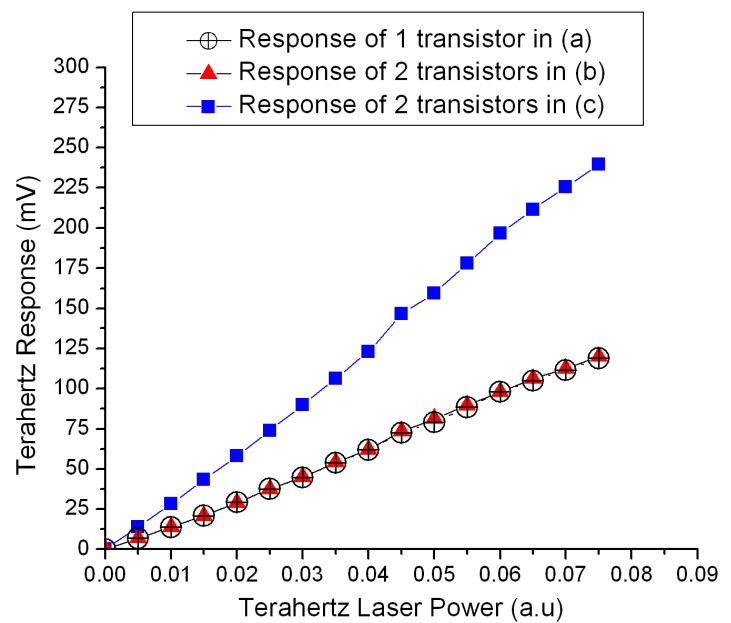

(d)
Fig. 3. Circuit schematics showing dc operating points for the three testing configurations of the two transistors test structure: (a) single transistor operated in saturation region, (b) one transistor operated in linear region and the other operated in saturation region, and (c) both transistors operatd in saturation region. The measured responsivities for the three cases are presented in (d).

According to this theory, we expect no response from symmetrical HEMT structures, when overdamped plasma waves of the same amplitude are excited at both sides of the symmetrical channel. We proved this experimentally: no response was detected without dc current flow in a symmetrical transistor (compare to [23]). The flow of dc current in this transistor creates asymmetry in boundary conditions at source and drain, asymmetry for propagation of plasma waves, and this asymmetry enhances plasma wave detection in the device [21]. In the saturation mode, asymmetry across the transistor channel increases and consequently the nonresonant $\mathrm{THz}$ response increases. In the linear region, the asymmetry across transistor channel is very small and in turn the transistor detects a very weak $\mathrm{THz}$ response. This explains the result of Fig. 3(d) in which the response from a single transistor operating in 
saturation region (Fig. 3(a)) is identical to the response of two transistors connected in series (Fig. 3(b)) where one is operating in linear region and the other is operating in saturation region.

Figure 4 shows the dependence of $\mathrm{THz}$ response on the second gate $\left(g_{2}\right)$ bias voltage while keeping the first gate $\left(g_{1}\right)$ bias voltage at a lower fixed value for the circuit shown in Fig. 3(b). By increasing the bias voltage of the second transistor gate, we tuned the first transistor from linear region to saturation region while keeping the second transistor operating in saturation region. Our experimental result indicates that the response increases linearly when the first transistor goes from the linear region into the saturation region. The overall response almost saturates when both transistors operate in saturation region. This result implies that the response of each transistor is independent, and the overall response of the circuit is the summation of both transistors' responses.

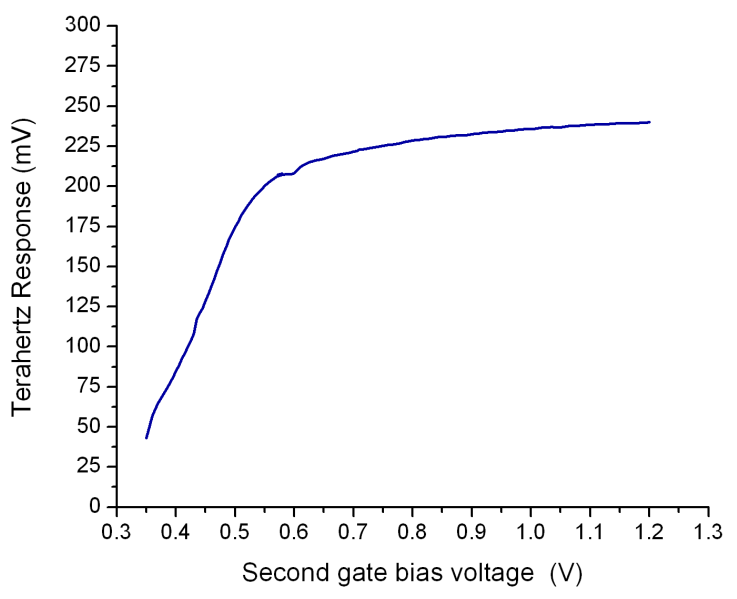

Fig. 4. The measured terahertz response versus second gate $\left(g_{2}\right)$ bias voltage while the first gate $\left(g_{1}\right)$ bias voltage was fixed at $0.44 \mathrm{~V}$ for the circuit configuration shown in Fig. 3(b).

Figure 5 shows the measured $\mathrm{THz}$ responsivities of one transistor and up to four transistors connected in series when all of them are operating in saturation region. In order to detect the maximum responsivity from the chain of four transistors in series, we biased them with $30 \mathrm{~V}$ through a $100 \mathrm{~K}$ load resistor. However, we were not able to adjust the gate-source biasing of the third and fourth transistor to be exactly similar to the other two transistors due to their loading effect. This explains our results in Fig. 5 in which the $\mathrm{THz}$ response of three transistors was found to be $2.92 \mathrm{x}$ that of one transistor, while the $\mathrm{THz}$ response of four transistors was found to be $4.1 \mathrm{x}$ that of one transistor. Still, these results show that the overall response is approximately proportional to the number of the transistors connected in series.

Since the feature size of each transistor is much smaller than the wavelength, a large number of transistors can be stacked in the focal spot of the incident $\mathrm{THz}$ radiation and a higher response can be detected. An even larger enhancement is expected when the dimension of the transistor chain would exceed the $\mathrm{THz}$ wavelength as predicted in $[17,18]$. Connecting many transistors in series for $\mathrm{THz}$ detection as described here offers another important advantage by improving the signal-tonoise ratio (SNR). SNR will be improved by a factor of square root of the number of connected transistors. This advantage makes the series connection more preferable than utilization of a simple amplifier circuit for these types of $\mathrm{THz}$ plasma wave detectors.

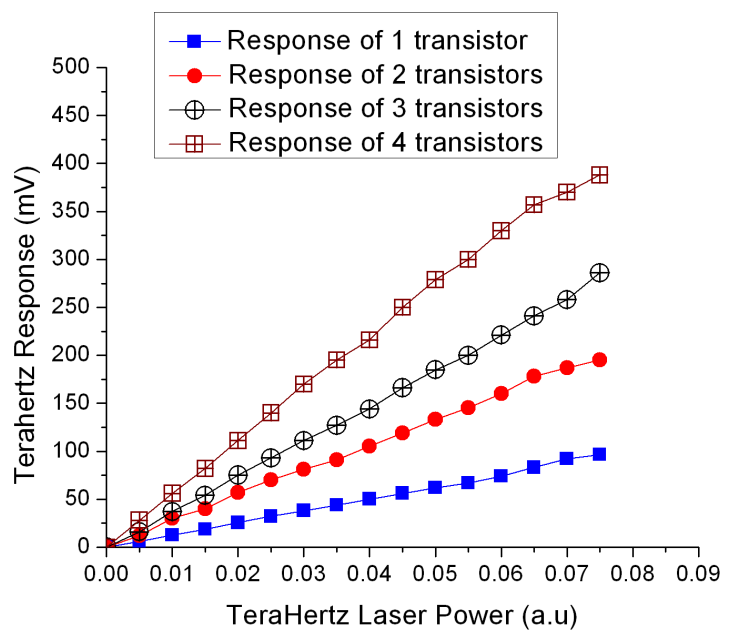

Fig. 5. Measured responsivities of one, two, three, and four transistors connected in series. All transistors were operating in saturation region.

\section{CONCLUSION}

We have presented an enhanced nonresonant detection of terahertz radiation using multiple GaAs HEMTs connected in series. We proved experimentally using our fabricated symmetrical test structures of two and four transistors that the overall response of series connection is equal to the sum of $\mathrm{THz}$ responses of each transistor. Using such transistor chains for $\mathrm{THz}$ detection allows for novel circuit solution for modulation and mixing of $\mathrm{THz}$ response and for imaging applications.

\section{REFERENCES}

[1] Yunqing Chen, Haibo Liu, Yanqing Deng, Dmitry Veksler, Michael Shur, X.-C. Zhang, Dunja Schauki, Michael J. Fitch, and Robert Osiander, "Spectroscopic characterization of explosives in the far infrared region," Proceedings of SPIE, vol. 5411, no. 1, pp. 1-8, October 2004.

[2] H. Liu, G. Plopper, S. Earley, Y. Chen, B. Ferguson, and X.-C. Zhang, "Sensing Minute Changes in Biological Cell Monolayers with THz Differential Time-domain Spectroscopy," Biosensor and Bioelectronics, vol. 22, no. 6, pp. 1075-1080, January 2007.

[3] S. P. Mickan and X.-C. Zhang, "T-ray Sensing and Imaging," International Journal of High Speed Electronics and Systems, vol. 13, no. 2, pp. 601-676, June 2003.

[4] M. Kroug, S. Cherednichenko, H. Merkel, E. Kollberg, B. Voronov, G. Goltsman, H. W. Huebers, H. Richter, "NbN hot electron bolometric mixers for terahertz receivers," IEEE Transactions on Applied Superconductivity, vol. 11, no. 1, pp. 962-965, March 2001. 
[5] S. Barbieri, J. Alton, H. E. Beere, E. H. Linfield, D. A. Ritchie, S. Withington, G. Scalari, L. Ajili, and J. Faist, "Heterodyne mixing of two far-infrared quantum cascade lasers by use of a point-contact Schottky diode," Optics Letters, vol. 29, no. 14, pp. 1632-1634, July 2004.

[6] J.-Q. Wang, P. L. Richards, J. W. Beeman, and E. E. Haller, "Stressed photoconductive detector for far infrared space applications," Applied Optics, vol. 26, no. 22, pp. 4767-4771, November 1987.

[7] Y. Deng, M.S. Shur, R. Gaska, G.S. Simin, M.A. Khan, and V. Ryzhii, "Millimeter wave emission from GaN high electron mobility transistor," Applied Physics Letters, vol. 84, no. 1, pp. 70-72, January 2004.

[8] W. Knap, J. Lusakowski, T. Parenty, S. Bollaert, A. Cappy, V.V. Popov, and M.S. Shur, "Terahertz emission by plasma waves in $60 \mathrm{~nm}$ gate high electron mobility transistors," Applied Physics Letters, vol. 84, no. 13, pp. 2331-2333, March 2004.

[9] W. Knap, Y. Deng, S. Rumyantsev, J.-Q. Lu, M.S. Shur, C.A. Saylor, and L.C. Brunel, "Resonant detection of subterahertz radiation by plasma waves in a submicron field-effect transistor," Applied Physics Letters, vol. 80, no. 18, pp. 3433-3435, May 2002.

[10] W. Knap, V. Kachorovskii, Y. Deng, S. Rumyantsev, J.-Q. L, R. Gaska, M. S. Shur, G. Simin, X. Hu and M. Asif Khan, C. A. Saylor, L. C. Brunel, "Nonresonant detection of terahertz radiation in field effect transistors," Journal of Applied Physics, vol. 91, no. 11, pp. 9346-9353, June 2002.

[11] W. Knap, F. Teppe, Y. Meziani, N. Dyakonova, J. Lusakowski, F. Boeuf, T. Skotnicki, D.K. Maude, S. Rumyantsev, and M.S. Shur, "Plasma wave detection of sub-terahertz and terahertz radiation by silicon fieldeffect transistors," Applied Physics Letters, vol. 85, no. 4, pp. 675-677, July 2004.

[12] N. Pala, F. Teppe, D. Veksler, Y. Deng, M.S. Shur, and R. Gaska, "Nonresonant detection of terahertz radiation by silicon-on-insulator MOSFETs," Electronics Letters, vol. 41, no. 7, pp. 447-449, March 2005.

[13] F. Teppe, W. Knap, D. Veksler, M.S. Shur, A.P. Dmitriev, V. Yu. Kachorovskii, and S. Rumyantsev, "Room-temperature plasma waves resonant detection of sub-terahertz radiation by nanometer field effect transistor," Applied Physics Letters, vol. 87, no. 5, pp. 052107-3, August 2005.
[14] V. Yu. Kachorovskii, and M.S. Shur, "Field effect transistor as ultrafast tunable detector of terahertz radiation," Solid State Electronics, vol. 52, no. 2, pp. 182-185, February 2008.

[15] Dmitry Veksler, Andrey Muravjov, William Stillman, Nezih Pala, and Michael Shur, "Detection and Homodyne Mixing of Terahertz Gas Laser Radiation by Submicron GaAs/AlGaAs FETs," in Abstracts of IEEE Sensors Conference, Atlanta, GA, USA, pp. 229-230, October 2007.

[16] R. Weikle, J. Lu, M.S. Shur, M.I. Dyakonov, "Detection of Microwave Radiation by Electronic Fluid in High Electron Mobility Transistors," Electronics Letters, vol. 32, no. 23, pp. 2148-2149, November 1996.

[17] V.V. Popov, G.M. Tsymbalov, and M.S. Shur, "Plasma Wave Instability and Amplification of Terahertz Radiation in Field-Effect-Transistor Arrays," Journal of Physics: Condensed. Matter, vol. 20, no. 38, pp. 384208-6, September 2008.

[18] V.V. Popov, M.S. Shur, G.M. Tsymbalov, and D.V. Fateev, "Higherorder plasmon resonances in GaN-based field-effect transistor arrays," International Journal of High Speed Electronics and Systems, vol. 17, no. 3, pp. 557-566, September 2007.

[19] J.-Q. Lu and M.S. Shur, "Terahertz detection by high electron mobility transistor: Enhancement by drain bias," Applied Physics Letters, vol. 78, no. 17, pp. 2587-2588, April 2001.

[20] M. Dyakonov and M.S. Shur, "Shallow water analogy for a ballistic field effect transistor: New mechanism of plasma wave generation by dc current," Physical Review Letters, vol. 71, no. 15, pp. 2465-2468, October 1993.

[21] D. Veksler, F. Teppe, A. P. Dmitriev, V. Y. Kachorovskii, W. Knap, and M. S. Shur, "Detection of terahertz radiation in gated two-dimensional structures governed by dc current," Physical Review B, vol. 73, no. 12, pp. 125328-125338, March 2006.

[22] W. Stillman, M. S. Shur, D. Veksler, S. Rumyantsev, and F. Guarin, "Device loading effects on nonresonant detection of terahertz radiation by silicon MOSFETs," Electronics Letters, vol. 43, no. 7, pp. 422-423, March 2007.

[23] D. Veksler, A. V. Muraviev, T. A. Elkhatib, K. N. Salama, and M. Shur, "Plasma wave FET for sub-wavelength THz imaging," Proceedings of IEEE ISDRS 2007, College Park, MD, USA, pp. 1-2, December 2007. 